\title{
Perioperative Pain Management: Present outlook
}

The individuals rights in the United States, and the rise of democratic states, has created an environment in which individual pursuit of better health care, including pain relief, became explicit goals in the civilized world. ${ }^{1}$ The 'right to pain relief has now multidimensional foundations in the developed world. ${ }^{2}$ Knowingly, acute pain is a universal phenomenon. All emergency and elective surgery, severe medical illness, trauma, childbirth, burns, natural calamities, war and torture, all contribute to its burden. In many countries political conflict, social dislocation, and inadequate availability of analgesics conspire to make the relief of acute pain sporadic at best. Effective and efficient pain control, however, is an ethical responsibility and moral obligation of a caring physician, whether working in the developed or developing world.

Perioperative pain management commences from the preoperative assessment period and continues as adequate pain control during surgery and a well planed post operative pain control, both during early postoperative phase and in convalescence period, using appropriate analgesic techniques, customized and rationalized for every individual patient. There should be objective analysis of the post operative pain control techniques and patient's right to remain pain free should not be compromised at any cost.

All surgical procedures are followed by pain, which may amplify metabolic, endocrine responses, autonomic reflexes, nausea, vomiting, acute muscular spasm, paralytic ileus, respiratory insufficiency, and may delay not only the restoration of functions, but greatly affect the psychological well being of the patients. Post-operative hypoxemia, immunosuppression, muscle catabolism and wasting, thromboembolic complications, cardiac events, post operative infections, gastrointestinal and even sleep disturbance are directly related with post-operative pain control. ${ }^{3}$ Post-operative fatigue, immobility, muscle wasting, nutritional impairments resulting from poorly managed post-operative pain, adversely affect convalescence period and the speed of recovery. ${ }^{4}$ The recent systematic review of the pain literature by Liu and $\mathrm{Wu}$ examined the effect of the postoperative analgesic techniques on the incidence of complications after surgery. The authors reanalyzed 18 meta-analyses and 10 systematic reviews, and concluded that post operative morbidity has direct relationship with post-operative pain control and convalescence. ${ }^{5}$ Reviewing the available literature from the last two decade greatly emphasized the importance of efficient and timely post operative pain control to reduce the post operative morbidity and mortality. Unrelieved pain control after surgery increases heart rate, systemic vascular resistance and circulating catecholamines, placing susceptible patients at risk for myocardial ischemia, stroke, bleeding and other complications. Unrelieved acute pain commonly elicits pathophysiologic neural alterations, including peripheral and central nociceptive sensitization, that evolve into chronic pain syndromes. ${ }^{6}$ Apart from the above reasons medical text provides us exhaustive data and authorities are fully convinced to effectively and efficiently manage post operative pain due to ethical, cultural, and legal concerns. ${ }^{7}$ Attitudes of the societies toward pain relief during surgery and childbirth illustrate the complex interactions between cultural concepts of pain, pain relief, and social behavior. The treating physician must also understand and tailor his pain management strategy and tools according to the individual patient, his/her wishes, understandings, social and cultural beliefs, not merely essential understanding of pain pathophysiology, methods of pain control and their clinical implications. Comprehensive pain management definitely relates positive impact on the outcome of surgery. ${ }^{8}$

Good pain control after surgery is important to prevent negative outcomes such as tachycardia, hypertension, myocardial ischemia, decrease in alveolar ventilation, and poor wound healing. Exacerbations of acute pain can lead to neural sensitization and release of mediators both peripherally and centrally. Clinical wind up occurs from the processes of N-Methyl DAspartate (NMDA) activation, wind up central sensitization, long-term potentiation of pain, and transcription-dependent sensitization. Advances in the knowledge of molecular mechanisms have led to the development of multimodal analgesia and new pharmaceutical products to treat postoperative pain. The new pharmacological products to treat postoperative pain include extended-release epidural morphine 
(Depodur) and analgesic adjuvants such as capsaicin, ketamine, gabapentin, pregabalin dexmetomidine, and tapentadol. Newer postoperative patient-controlled analgesia (PCA) in modes such as intranasal, regional, transdermal, and pulmonary presents another interesting avenue of development. ${ }^{19,10}$

Surgical tissue injury provokes a neuroendocrine stress response and inflammation. ${ }^{11}$ The neuroendocrine response can be moderated by regional or neuraxial anesthesia. It is also likely that inflammation contributes to acute postoperative pain. A variety of anti-inflammatory medications including lidocaine, selective cyclooxygenase- 2 inhibitors, and other nonsteroidal anti-inflammatory drugs have thus been used in attempts to reduce surgical pain. ${ }^{12}$ The ultimate antiinflammatory drugs, however, are steroids. To the extent that inflammatory mechanisms contribute to postoperative pain, one might expect that preoperative or intraoperative steroid administration would ameliorate postoperative pain. ${ }^{13}$ Corticosteroids also inhibit expression of cytokine genes and release of proinflammatory enzymes, bradykinin, and neuropeptides from injured nerve terminals - all of which also worsen pain. As might thus be expected, many studies have evaluated the effects of steroid administration on surgical pain. "Dexamethasone ameliorates acute postoperative pain ... [but] what remains unclear is the risk-benefit ratio."

Local infiltration analgesia after hip and knee arthroplasty, transversus abdominis plane block after abdominal operations and local application of capsaicin lend some promise. ${ }^{14}$

Pharmacologic advances in anesthesia over the last decade have focused on drug safety, shorter durations of action, reversibility, and ease of administration. This is reflective of major changes in the focus of patient care from inpatient to outpatient settings as well as from available risk management data that support the investigation of these new drugs. Several of the newer synthetic narcotics, such as remifentanil, alfentanil and sufentanil, have replaced other narcotics formerly used in anesthesia practice, such as meperidine and morphine, primarily because of their short action and lack of significant side effects. As research continues, new drugs will be incorporated into the practice of anesthesia, ones that will promote rapid uptake, low toxicity, intense analgesia, easy reversibility, shorter durations, and fewer side effects. One measure of success relative to pharmacologic development in anesthesia is the recent and dramatic decreases in patient morbidity and mortality figures over the last decade. This attests to the rapid growth and development of not only improved patient monitoring systems but also newly improved "analgesics and local anesthetics."

The subspecialty of Regional Anesthesia and Acute Pain Medicine is rapidly changing, necessitating frequent updates in pharmacology, techniques and practice protocols. The introduction of ultrasound in the clinical practice of regional anesthesia has resulted in an unprecedented change in the specialty as well as in a growth of the interest for regional anesthesia. A number of highly efficacious peripheral nerve block techniques are now available to provide superb surgical anesthesia and postoperative analgesia in patients undergoing a wide variety of surgical procedures. Judiciously and skillfully performed nerve blocks facilitate pain management, fasttracking, promote early mobilization, decrease hospital stay, reduce unanticipated hospital admission and reduce overall health care costs. Now comprehensive updates on the latest developments in regional anesthesia and acute pain management are available and have evolved into current regional anesthesia techniques and practice protocols. In addition, a comprehensive range of hands-on workshops should be arranged on regular basis as here in the United States to provide attendees an opportunity to acquire or improve their skills in a number of regional anesthesia techniques.

Some papers published in present issue of this journal aim to review and discuss developments within the field various adjuncts, systemic analgesics and local/regional anesthetic methods for management of postoperative pain, based on evidence from randomized, clinical trials published within the last 5 years, but there is still a lack of well-performed RCTs to draw any firm conclusions.

Procedure-specific analgesic combinations within welldefined rehabilitation paradigms should be explored further to reduce adverse effects associated with the use of conventional analgesic treatment protocols, and to improve postoperative outcome.

Javaid A. Zargar, M.D.

Department of Anesthesiology

Elmhurst Hospital and Medical Center, New York

Email: zargarja@nychhc.org

Abdul Qayoom Lone, M.D.

Additional Professor,

Department of Anesthesiology \& Critical Care,

Sher-i-Kashmir Institute of Medical Sciences, Srinagar, Kashmir

(J\&K) India - 190011

Email: drloneaq@hotmail.com

\section{References}

1. Kehlet H. Post operative pain what is issue. Br J Anaesth 1994;72:375-378.

2. Brennan F, Carr BD, Cousins MJ. Pain Management as a fundamental human right. Anesth Analg 2007;105: 205-21.

3. Kehlet H, Dahl JB. Anaesthesia, surgery and challenges in postoperative recovery. Lancet 2003;362:1921-8.

4. Paul F. White, Henrik Kehlet. Postoperative pain management and patient outcome: Time to Return to Work. A\& A 2007;104(3):487-489.

5. Liu SS, Wu CL. Effect of postoperative analgesia on postoperative complications: a systematic update of the evidence. Anesth Analg 2007;104:689-702.

6. Perkins FM, Kehlet H. Chronic pain as an outcome of 
surgery. A review of predictive factors. Anesthesiology 2000;93:1123-33.

7. Apfelbaum JL, Chen C, Mehta SS, Gan TJ. Postoperative pain experience: results from a national survey suggest postoperative pain continues to be undermanaged. Anesth Analg 2003; 97:534-540.

8. Breivik H. Postoperative pain management: why is it difficult to show that it improves outcome? Eur J Anaesthesiol 1998;15(6):748-751.

9. Carr D, Goudas L. Acute pain. Lancet 1999; 353(9169): 2051-2058.

10. Breivik H, Stubhaug A. Management of acute postoperative pain: still a long way to go! Pain 2008;137(2): 233-234.
11. Udelsman R, Holbrook N. Endocrine and molecular responses to surgical stress. Curr Probl Surg 1994; 31: 653-720.

12. Kohl BA, Deutschman CS. The inflammatory response to surgery and trauma. Curr Opin Crit Care 2006;12: $325-32$.

13. De Oliveira GS Jr, Almeida MD, Benzon HT, and McCarthy RJ. Perioperative single dose systemic dexamethasone for postoperative pain: A meta-analysis of randomized controlled trials. Anesthesiology 2011;115: 575-88.

14. Bagry H, de la Cuadra Fontaine JC, Asenjo JF, Bracco D, Carli F. Effect of a continuous peripheral nerve block on the inflammatory response in knee arthroplasty. Reg Anesth Pain Med 2008; 33:17-23. 